\title{
Performance of the LHCb Silicon Tracker in pp Collisions at the LHC
}

\author{
Mark Tobin \\ Physik Institut der Universität Zürich, Zürich, Switzerland \\ On behalf of the LHCb Silicon Tracker Group ${ }^{\dagger}$
}

\author{
M. Needham, A. Bay, F. Blanc, M.O. Bettler, J. Bressieux, G. Conti, F. Dupertuis, V. Fave, R. Frei, N. Gauvin, \\ G. Haefeli, A. Keune, J. Luisier, R. Muresan, T. Nakada, L. Nicolas, M. Knecht, C. Potterat, O. Schneider, \\ M. Tran \\ École Polytechnique Fédérale de Lausanne \\ O. Aquines Gutierrez. C. Bauer, M. Britsch, W. Hofmann, F. Maciuc, M. Schmelling, H. Voss \\ Max Planck Institut für Kernphysik, Heidelberg \\ J. Anderson, A. Buechler, A. Bursche, N. Chiapolini, M. De Cian, C. Elsasser, C. Salzmann, S. Saornil, \\ S. Steiner, O. Steinkamp, U. Straumann, J. van Tilburg, M. Tobin, A. Vollhardt \\ Physik-Institut der Universität Zürich \\ B. Adeva, A. Pazos Alvarez, D. Esperante, A. Gallas, E. Pérez Trigo, P. Rodríguez Pérez, J. Saborido, P. Vázquez \\ University of Santiago de Compostela \\ V. Iakovenko, O. Okhrimenko, V. Pugatch \\ National Academy of Sciences, Institute for Nuclear Research, Kiev.
}

\begin{abstract}
The LHCb detector is designed to study the decays of B-mesons in proton-proton collisions at the Large Hadron Collider (LHC). The detector is a single arm forward spectrometer with excellent tracking and particle identification capabilities. The Silicon Tracker (ST) consists of two detectors both of which are constructed from silicon micro-strip detectors with long readout strips. The performance of these detectors in the first high energy collisions at the LHC is presented here. The data collected in 2010 has been used to determine the optimal time and spatial alignment of the detectors. A detailed study has also been made of the intrinsic detector efficiency and resolution. The latest results on detector performance will be shown and compared to the expectation from previous measurements and the simulation of the detector.
\end{abstract}

\section{INTRODUCTION}

The LHCb experiment [2] is a single arm spectrometer designed to study heavy flavour physics in decays of Bmesons. It will constrain the parameters of the CKM matrix[3] by measuring $\mathrm{CP}$-violation and probe for physics beyond the Standard Model by studying rare decays. The detector covers polar angles in the range 15 to $300 \mathrm{mrad}$ and exploits the fact that both b-quarks are produced in the same forward cone at the LHC.

The LHCb Silicon Tracker is a silicon micro-strip detector with a sensitive area of approximately $12 \mathrm{~m}^{2}$ and a total of $272 \mathrm{k}$ readout channels. It consists of two detectors: the Tracker Turicensis (TT), a $150 \mathrm{~cm}$ wide and $130 \mathrm{~cm}$ high tracking station covering the full LHCb acceptance upstream of the LHCb magnet; and the Inner Tracker (IT) which covers

\footnotetext{
$\dagger$ Submitted to the conference record for the 2010 IEEE Nuclear Science Symposium, Knoxville, Tennessee, USA, 30 Oct - 6 Nov 2010
}

approximately $1 \%$ of the total acceptance in a $120 \mathrm{~cm}$ by $40 \mathrm{~cm}$ cross-shaped region in the centre of three planar tracking stations downstream of the magnet.

The Tracker Turicensis has four detection layers orientated at $\left(0^{\circ},+5^{\circ},-5^{\circ}, 0^{\circ}\right)$ with respect to the y-axis. The detector is constructed from $500 \mu \mathrm{m}$ thick p-on-n sensors with a pitch of $183 \mu \mathrm{m}$. Sensors are bonded together to provide readout sectors with 1,2, 3 or 4 sensors depending on their position relevative to areas of higher particle flux. This leads to four different length readout strips (up to $37 \mathrm{~cm}$ ). There are 280 readout sectors in the TT and a total of 143600 readout channels. The TT is shown in figure 1.

The three Inner Tracker stations each consist of four independent boxes arranged around the beam pipe. Each box contains four detection layers at $\left(0^{\circ},+5^{\circ},-5^{\circ}, 0^{\circ}\right)$ with respect to the $y$-axis. The ladders either side of the beam pipe use $410 \mu \mathrm{m}$ thick sensors and have a length of $22 \mathrm{~cm}$ while those above and below are $11 \mathrm{~cm}$ long and $320 \mu \mathrm{m}$ thick. There are 336 readout sectors in IT and a total of $129 \mathrm{k}$ readout channels. The IT is shown in figure 2.

The number of working channels in the TT and IT at the time of the conference was $99.45 \%$ and $98.53 \%$ respectively.

\section{Commissioning Without BeAm}

The installation of the detectors was completed in summer 2008. There followed an extensive commissioning campaign before the first proton-proton collisions in 2009. The detector was run both in standalone tests and in $\mathrm{LHCb}$ cosmic runs which allowed to test the robustness of the readout chain and to perform a coarse time alignment of the detector. The 


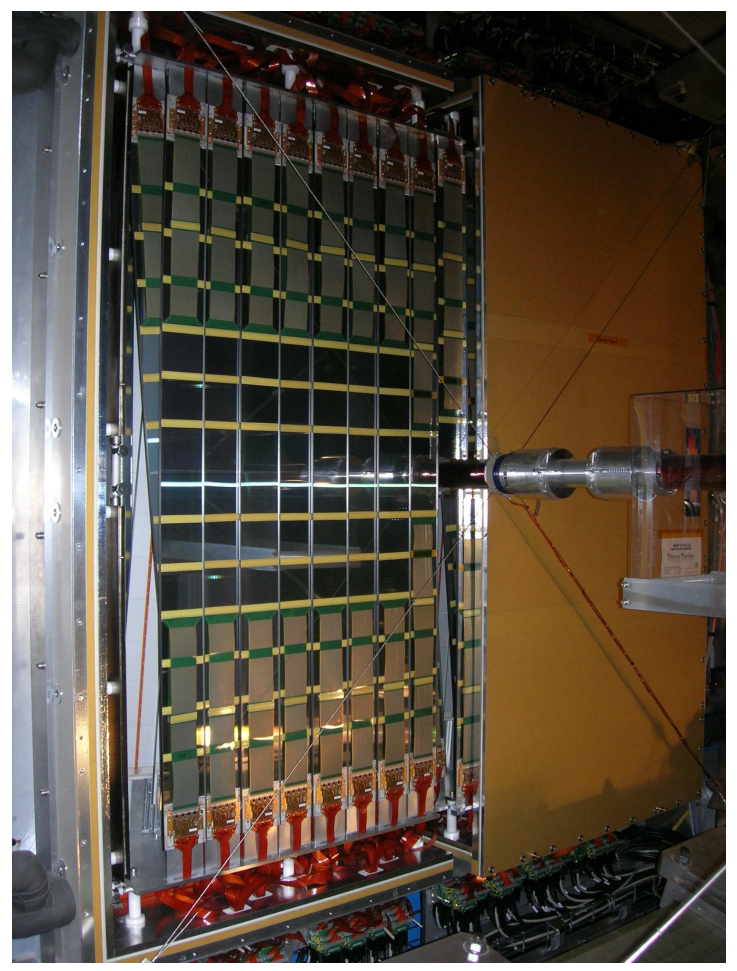

Fig. 1. The Tracker Turicensis after installation at LHCb.

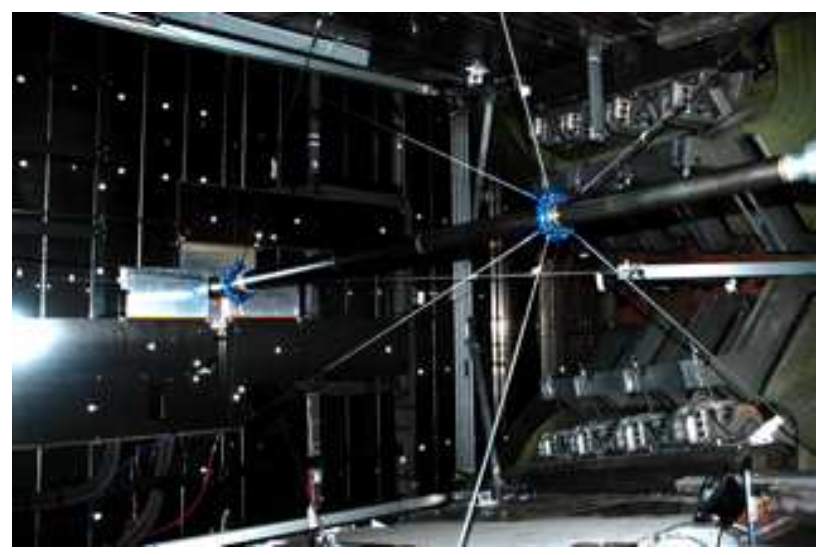

Fig. 2. The Inner Tracker after installation at LHCb.

forward geometry of LHCb meant that only three tracks were reconstructed traversing all three IT stations in 2.6 million triggers taken during the $\mathrm{LHCb}$ cosmic runs.

In addition, further tests were made without colliding beams using data taken during the LHC synchonisation tests in 2008 and 2009. Protons were extracted from the SPS and dumped onto a beam stopper ("TED") located $350 \mathrm{~m}$ away from $\mathrm{LHCb}$ resulting in a stream of $10 \mathrm{GeV}$ muons passing through $\mathrm{LHCb}$. This made it possible to perform a first time and spatial alignment of the detector with tracks.

\section{COMMISSIONING WITH BEAM}

\section{A. Time Alignment}

The first proton-proton collision events were used to time align the detector with respect to the LHC collisions. It is important to ensure that the trigger and control signals are synchronised across the whole of LHCb. The internal time alignment was made to account for different length cables and different time of flight for particles passing each station. The optimal delay settings were determined from a scan using runs taken where the delay between the sampling time and the trigger was varied in $6.5 \mathrm{~ns}$ steps. The most probable value (MPV) was determined by fitting a Landau convolved with a Gaussian to the distribution of the charge for groups of ladders. The peak of the pulse shape was determined by fitting the expected front-end response to the distribution of the MPV versus the delay. An example is given in figure 3. After this procedure, the detector was time aligned to better than $1 \mathrm{~ns}$ precision.

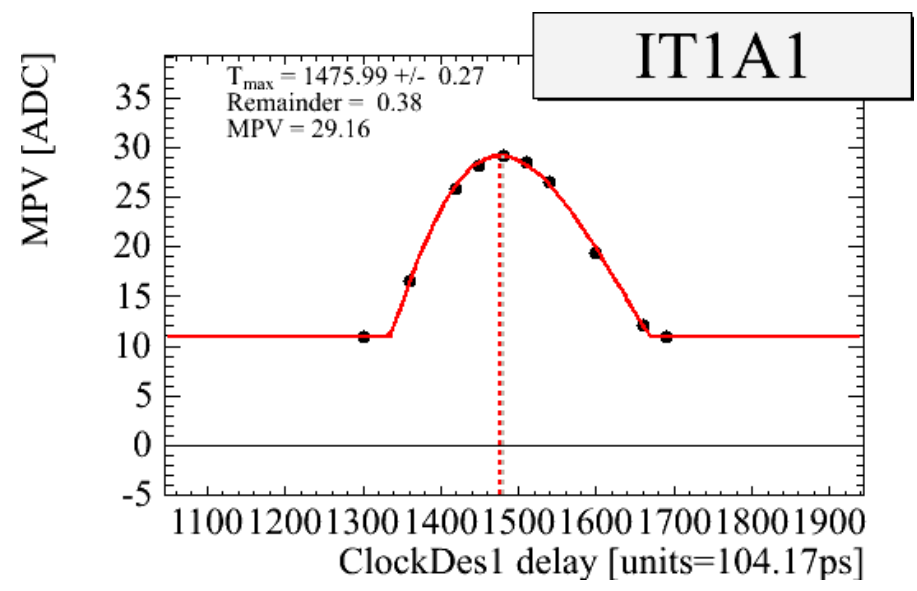

Fig. 3. The Most Probable Values obtained from fits to the ADC distributions for different trigger delays for a set of short ladders in IT. The optimal delay is extracted by fitting the expected pulseshape of the front-end chip to the distribution.

The signal to noise ratio $(\mathrm{S} / \mathrm{N})$ was determined after the time alignment procedure using tracks reconstructed with momentum greater than $5 \mathrm{GeV}$. The $\mathrm{S} / \mathrm{N}$ for the TT was found to be in the range 12 to 15 and is shown in figure 4 for the different strip capacitances. The long and short ladders in IT have a $\mathrm{S} / \mathrm{N}$ of 16.5 and 17.5 respectively. The distribution of the $\mathrm{S} / \mathrm{N}$ for all ladders in the IT is shown in figure 5 . The values obtained are within 10-20\% of those expected from previous measurements[4]

\section{B. Spatial Alignment}

The first collision data was used to make a spatial alignment of the detector. The procedure is based on a closed-form alignment method using tracks fitted with a Kalman filter and is described in [5]. The TT was aligned using (long) tracks which pass through the whole LHCb tracking system. The IT was aligned using long tracks but also using a standalone track reconstruction in the three tracking stations downstream of the magnet.

The unbiased residuals for hits on tracks are shown in figures 6 and 7 for TT and IT respectively. There is still a discrepency between the resolution expected from Monte Carlo and data and work is ongoing to resolve this. The remaining bias of the method can be estimated by looking at 


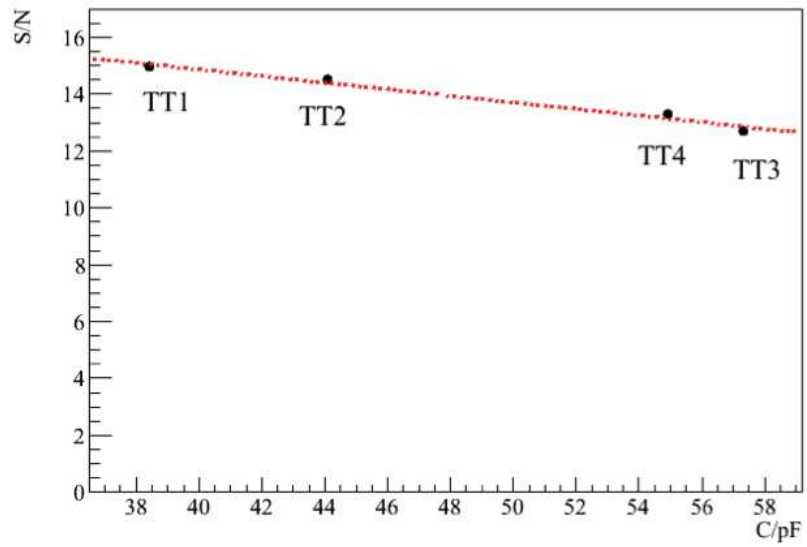

Fig. 4. Measured signal to noise ratio in TT as a function of readout sector capacitance.

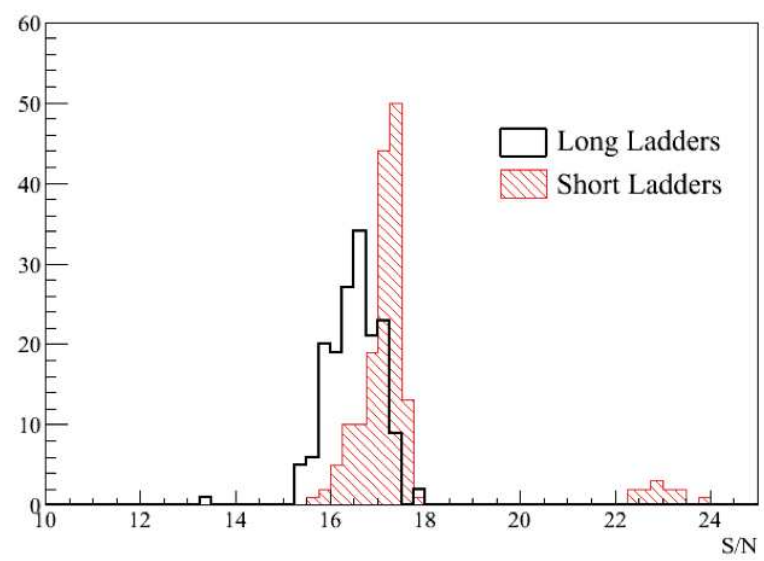

Fig. 5. Measured signal to noise ratio for all IT ladders. The second peak around 20 for the short ladders is due to the use of $410 \mu \mathrm{m}$ thick sensors during the construction.

the width of the distribution of the mean values of the unbiased hit residuals for each readout sector. It was found to be 17.7 and $11.1 \mu \mathrm{m}$ for TT and IT respectively.

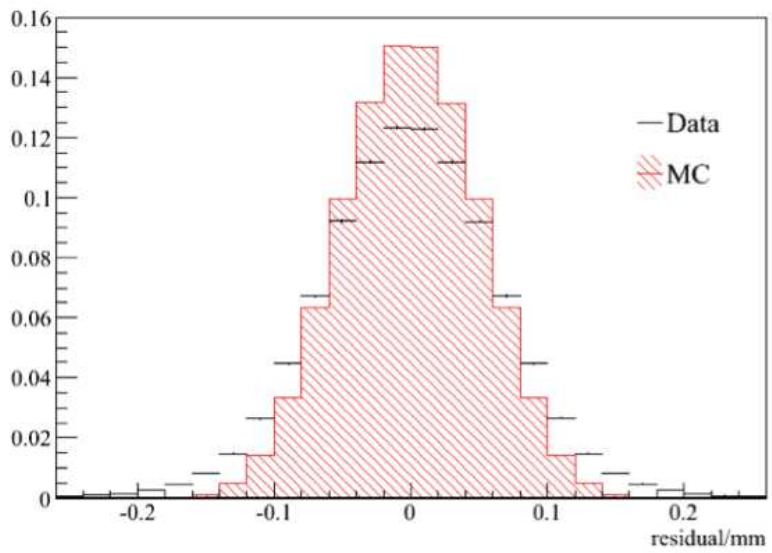

Fig. 6. Unbiased residual for TT clusters on tracks.

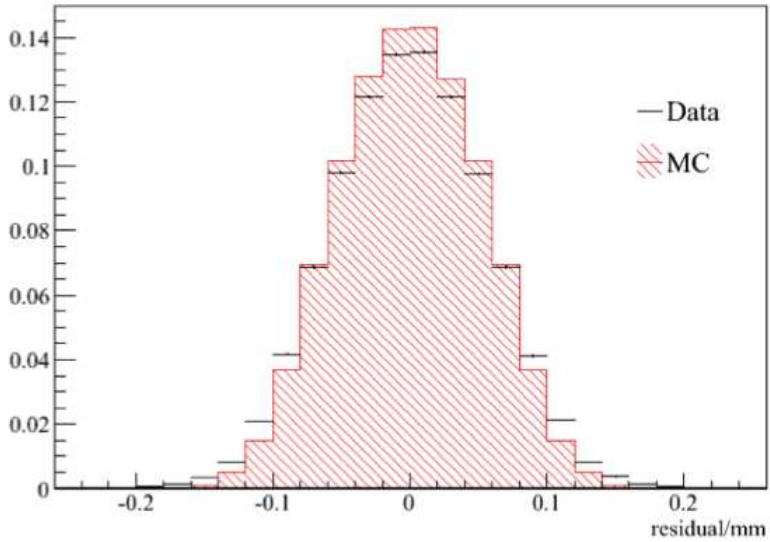

Fig. 7. Unbiased residual for IT clusters on tracks.

\section{Detector Performance}

The data collected from proton-proton collisions at $\sqrt{\mathrm{s}}=7 \mathrm{TeV}$ were used to study the detector performance and improve the modelling of the detector response in the simulation. This is described in the following sections.

\section{A. Hit Efficiency}

The intrinsic detector efficiency was measured using high momentum tracks $(\mathrm{P}>10 \mathrm{GeV})$. For each layer where a hit is expected, hits are searched for in a window around the track. The efficiency is defined as the ratio of the number of found hits to the number of expected hits. The efficiency was measured to be $99.3 \%$ and $99.65 \%$ for TT and IT respectively. The noise cluster rate was below $10^{-5}$.

\section{B. Charge Sharing}

The intrinsic charge sharing between two strips has been measured using the high energy collision data[6]. The cluster centre-of-gravity, $\eta^{1}$, is plotted as a function of the relative track interstrip fraction, $x$, and the effective charge sharing width is extracted by fitting an error function convolved with a Gaussian to this distribution. This is shown in figure 8. The width of the Gaussian gives the intinsic charge sharing width.

The intrinsic charge sharing width has been measured using tracks passing perpedicular to the sensor to be in the range 3.5 to $5.0 \mu \mathrm{m}$. This is lower than expected from previous measurements where the charge sharing width was found to be 11 to $33 \mu \mathrm{m}$ [7][4]. This discrepency is under investigation. As expected, the charge sharing width increases as a function of track angle and is shown in figure 9.

\section{Comparison with Simulation}

The data taken has been used to tune the simulation. The following effects were measured using collison data: the capacitive coupling (or cross talk) between the strips; the

${ }^{1} \eta=\frac{Q_{R}}{Q_{L}+Q_{R}}$ where $Q_{L}$ and $Q_{R}$ are the charge measured on the strips to the left and right of the track which created the two strip cluster. 


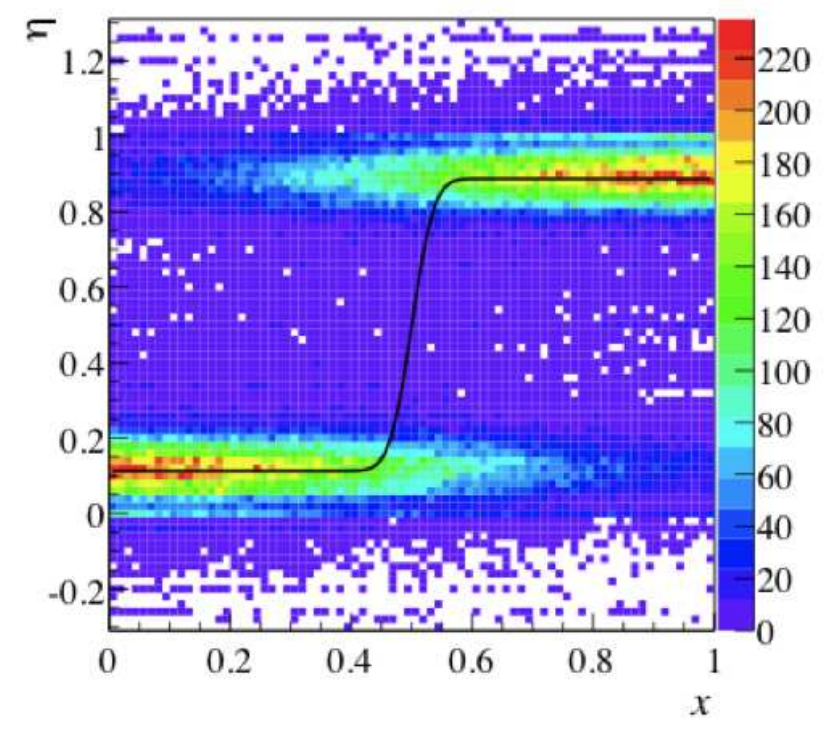

Fig. 8. Distribution of the cluster position, $\eta$, versus the relative track position, $x$. The charge sharing function obtained from a fit to the 2-D distribution is shown as the back curve.

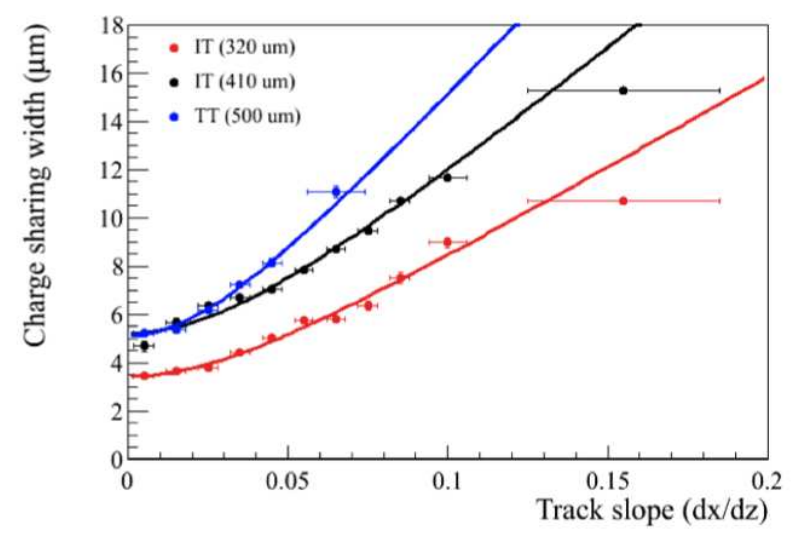

Fig. 9. Charge sharing width as a function of the track slope, dx/dz.

intrinsic charge sharing (c.f. section IV-B); tuning of the gain (conversion from electrons to ADC) for each sector. This is described in detail in [6]. The cluster size for TT is shown in figure 10 as a function of the track slope. The cluster size for long readout strips in the IT is shown in figure 11. The agreement between data and simulation is excellent.

\section{CONCLUSION}

The detector was installed by summer 2008. A programme of commission without beam followed to test the robustness of the readout chain. The fraction of the dector which is fully working is $99.45 \%$ and $98.53 \%$ for TT and IT respectively.

The first proton-proton collision data has been used to time align the dector with an accuracy of $1 \mathrm{~ns}$. The signal to noise ratio was found to be in the range 12 to 15 for different strip lengths in the TT. The long and short IT ladders have signal to noise ratios of 16.5 and 17.5 respectively.

Spatial alignment of the detector was performed. The hit resolution is worse in data compared to simulation and work

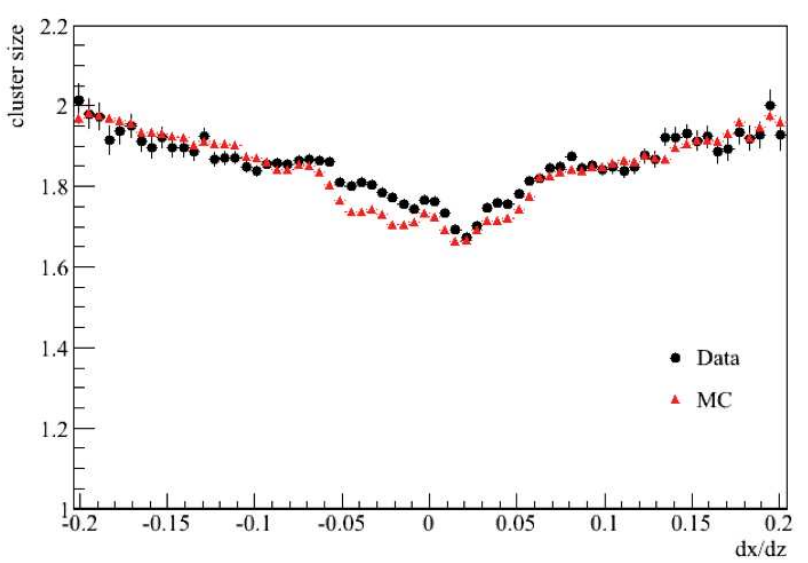

Fig. 10. Cluster size as a function of the track slope, $\mathrm{dx} / \mathrm{dz}$ for TT.

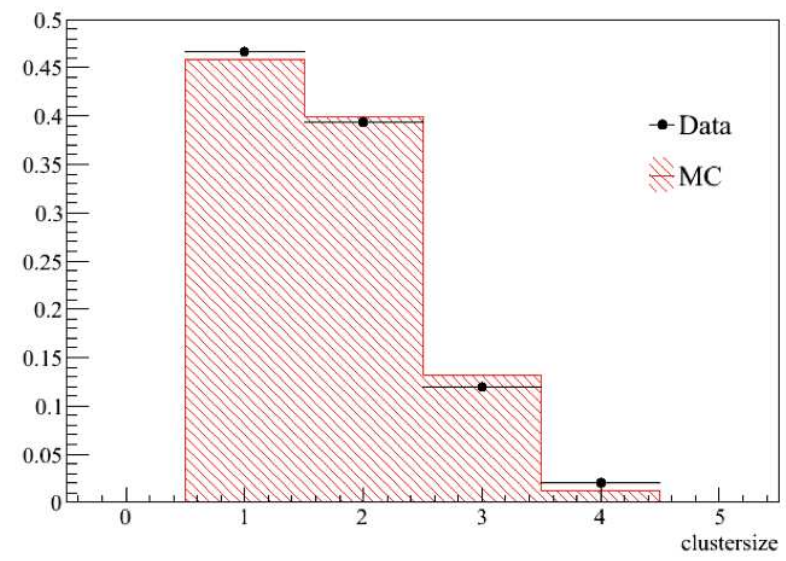

Fig. 11. Distribution of the cluster size for sectors with long readout strips in the IT.

is ongoing to understand this. The alignment precision is currently $11.1 \mu \mathrm{m}$ for IT compared to $17.7 \mu \mathrm{m}$ in TT.

The hit efficiency was measured using high momentum tracks to be $99.3 \%$ in TT and $99.65 \%$ in IT. The intrinsic charge sharing was measured using collision data and the effective charge sharing width was found to be in the range 3.5 to $5 \mu \mathrm{m}$ for different thickness sensor. Various other effects were also measured using data and used to tune the simulation of the detector. The comparison between data and the tuned simulation is excellent.

The LHCb Silicon Tracker is performing well.

\section{REFERENCES}

[1] LHCb Silicon Tracker Group, Performance of the LHCb Silicon Tracker in pp Collisions at the LHC, LHCb-PROC-2010-054 (2010).

[2] The LHCb Detector at the LHC, LHCb Collaboration, JINST 3 S08005 (2008).

[3] C. Amsler et al., Physics Letters B667, 1 (2008).

[4] M. Agari et al., LHCb-2002-058 (2002).

[5] W. Hulsbergen, Nucl. Inst. Meth. A600, 471-477 (2009).

[6] J. van Tilburg, Studies of the Silicon Tracker resolution using data, LHCbPUB-2010-016 (2010).

[7] C. Elsasser, Extention of the TT Test Stand with a Pulsed Focussed Infrared Laser, Bachelor Thesis, Physik Institut der Universität Zürich (2009). 